\title{
Mécanique de l'arbre sur pied : modélisation d'une structure en croissance soumise à des chargements permanents et évolutifs. 1. Analyse des contraintes de support
}

\author{
M Fournier 1, B Chanson 2, D Guitard 1, B Thibaut 2 \\ 1 CNRS, UMR CO23, INRA laboratoire de rhéologie du bois de Bordeaux, \\ domaine de l'Hermitage, BP 10, 33610 Cestas Gazinet; \\ 2 CNRS, URA 1214 laboratoire de mécanique et de génie civil, \\ université de Montpellier II, place Eugène-Bataillon, 34095 Montpellier Cedex 5, France
}

(Reçu le 5 février 1991; accepté le 3 juin 1991)

\begin{abstract}
Résumé - Une méthode d'analyse des contraintes mécaniques supportées par le bois de l'arbre sur pied est proposée, qui tient compte de la croissance secondaire. Elle est appliquée à l'étude des contraintes longitudinales engendrées par le poids propre supporté par la tige, chargement permanent à l'échelle de la croissance du tronc. Les distributions d'efforts ainsi calculées sont tout à fait inattendues : d'une part, les niveaux de contraintes sont très faibles partout à la périphérie de la tige, là où le bois est très jeune donc sollicité depuis peu de temps; d'autre part, la localisation à l'intérieur de la tige et le niveau de chargement des parties les plus tendues ou comprimées ne dépend pas seulement de l'état actuel observable mais de toute l'histoire de l'arbre. Les notions intuitives de "face tendue ou comprimée" sont donc à reconsidérer. L'illustration de ces conclusions est faite à travers plusieurs situations numériques réalistes : cas de l'arbre vertical parfaitement symétrique, d'un houppier qui s'excentre dans un plan fixe plus ou moins vite ou qui se redresse, d'un houppier qui s'excentre en changeant de direction.
\end{abstract}

mécanique de l'arbre / fonction de soutien / contrainte mécanique / croissance secondaire

Summary - Mechanics of standing trees: modelling a growing structure submitted to continuous and fluctuating loads. 1. Analysis of support stresses. A general analysis of mechanical stresses which develop in stems as the tree grows in weight and volume is presented and applied to the study of the distribution of longitudinal stresses due to the self weight supported. Compressive and bending loads, the main loads due to weight supported, are analysed using simple concepts of beam theory. The effect of radial growth is taken into account. Compared to the classical distribution of stresses in a non-growing initially straight cantilever beam and fully loaded at a given moment in time, the stress patterns so calculated are totally unconventional: on the one hand, stress values are very low everywhere at the stem surface where young wood has been loaded for a short time; on the other hand, the positions and values of maximal tensile or compressive stresses depend not only on the actual state but on the entire history of the tree. The intuitive concepts of "tensile or compressive face" must be reconsidered. These conclusions are shown by several realistic numerical simulations: in the case of the symmetrical straight tree (fig 3), near the pith where the wood is older, compressive stresses can be 3-6 times greater than the uniform stresses calculated from the standard distribution of the whole weight on the final cross-section. In the case of the tree which offsets its crown eccentrically in a fixed direction (fig 4), the greater stress is not at the surface: the more recent the offset is, the nearer to the surface is the position of greater stress. The case of the tree which straightens its eccentric crown in a fixed direction (fig 5), clearly shows that a 
tree which is straight at the present time can undergo quite high tensile or compressive stresses inside. In the case of the tree with an eccentric twisting crown (fig 6), the position of greater tensile or compressive stresses is not in the axis of the bending observed at present, but depends on the histo. ry of twisting.

\section{standing tree mechanics / support function / mechanical stress / secondary growth}

\section{INTRODUCTION}

Les termes de "face tendue ou comprimée" sont souvent employés par le forestier ou le technologue devant l'arbre sur pied assymétrique, incliné ou flexueux. Ces qualificatifs traduisent une observation géométrique dans l'état actuel de l'arbre, en termes de distribution locale d'efforts dus au support du houppier, en utilisant les concepts de la résistance des matériaux classique, qui analyse l'état d'une poutre déformée dans l'instant actuel, mais initialement droite. Or, de tels raisonnements ne décrivent pas la mécanique de l'arbre en croissance, qui n'est jamais une poutre droite verticale, et qui s'épaissit progressivement par l'activité cambiale, en même temps qu'il s'alourdit.

Un outil d'analyse des états mécaniques successifs de la section droite de la tige en croissance, soumise à des efforts de longue durée (c'est-à-dire dont les vitesses de variations sont d'un ordre de grandeur comparable à la vitesse de croissance radiale) est donc proposé, qui conduit à la remise en cause de l'idée traditionnelle d'une distribution de contraintes longitudinales linéaire sur la section droite, en tension maximale sur une face et en compression maximale sur la face opposée.

Ce travail est réalisé dans le cadre du programme "Mécanique de l'arbre" du groupement scientifique «rhéologie et mécanique du bois".

\section{MODES PRINCIPAUX DE SOLLICITATIONS LIÉS AU SUPPORT DES MASSES PAR UNE TIGE : COMPRESSION, FLEXION}

Considérons un arbre, supportant, au niveau d'un billon élémentaire, un poids $P$ dont le centre de gravité est A (fig 1). Avec les concepts classiques de la résistance des matériaux appliquée aux poutres (Laroze, 1980), le support de cette masse se traduit par différents modes de sollicitation, dépendant de la géométrie de l'arbre. Dans le but de retenir la description géométrique de complexité minimale apte à rendre compte de la réponse mécanique de l'arbre au niveau d'une section du fût, nous nous limiterons ici à une schématisation d'un tronc vertical droit avec houppier éventuellement excentré. Le poids $P$ se traduit alors par une sollicitation combinée de compression et de flexion pure, caractérisée par :

- un effort normal de compression :

$$
N=-P
$$

- un moment fléchissant d'intensité $P e$ dans le plan (z. $\delta)$, dont les composantes selon $x$ et $y$ sont :

$$
M^{F} x=-P \text { e } \sin \delta \text { et } M^{F} y=P e \cos \delta
$$

où $e$ et $\delta$ représentent respectivement l'intensité et la direction de l'excentricité du point $A$ dans un plan horizontal. 

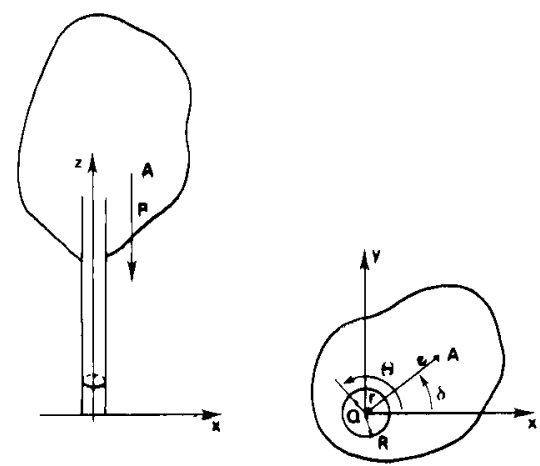

Flg 1. Données utiles à la schématisation. Le tronc supporte, au niveau d'une section droite, un poids $P$ dont le centre de poussée est $A$ de coordonnées polaires $(\theta, \delta)$ en projection dans le plan horizontal contenant la section droite. Un point quelconque de la section droite $Q$ est repéré par ses coordonnées polaires $(r, \theta)$.

Il est, de fait, indispensable d'introduire la notion d'excentricité du houppier et donc de sollicitation de flexion. En effet, d'une part il est clair que l'arbre ne présente pas forcément une symétrie intrinsèque de révolution (modèle architectural de Troll par exemple, où l'axe primaire est plagiotrope : Fisher et al, 1981; Edelin, 1989), et qu'il croît rarement dans un environnement isotrope. D'autre part, pour le mécanicien, l'élancement très important de la structure induit rapidement une prépondérance des effets de flexion sur ceux de compression (Fournier et al, 1990).

La schématisation mécanique pourrait aisément être complexifiée pour tenir compte de l'inclinaison et de la flexuosité du tronc (à condition de savoir les décrire géométriquement), elle montrerait alors d'éventuels effets de torsion.

\section{VARIATION DE L'ÉTAT MÉCANIQUE D'UNE TIGE ENTRE 2 INSTANTS $t$ ET $t+d t$, PROCHES DANS LE TEMPS}

La mécanique des solides analyse la variation d'état mécanique, entre deux instants initial et final, d'une structure, dont tous les points matériels ont une position fixée à l'instant initial, et que l'on soumet alors à un chargement. En référence à l'état initial, les déplacements des points matériels de la structure définissent un état de déformations, la répartition locale tridimensionnelle des efforts supportés dus au chargement définit un incrément de contraintes, dans l'état final (Guitard et al, 1989).

Nous nous proposons d'appliquer ces concepts généraux au problème suivant : quelle est, entre $t$ et $t+\mathrm{d} t$, la variation d'état mécanique, sous son poids propre, d'une tige de rayon $R$ à l'instant $t$, qui croît de $R$ à $R+\mathrm{d} R$ ?

Entre $t$ et $t+\mathrm{d} t$, la masse $P$ augmente de $\mathrm{d} P$, en même temps que son point d'application A se déplace ( $e$ devient $e+$ de, $\delta$ devient $\delta+\mathrm{d} \delta$ ). le chargement appliqué à l'instant $t$, entre $t$ et $t+\mathrm{d} t$, est donc en termes d'efforts intérieurs sur la section droite (par dérivation de (1)) :

- une compression :

$$
\mathrm{d} N=-\mathrm{d} P
$$

- un moment fléchissant de composantes :

$\mathrm{d} M F_{X}=-e \sin (\delta) \mathrm{d} P$

$-P \sin (\delta) \mathrm{de}-P e \cos (\delta) \mathrm{d} \delta$ $\mathrm{d} M{ }^{F} y=e \cos (\delta) \mathrm{d} P$

$+P \cos (\delta) \mathrm{de}-P e \sin (\delta) \mathrm{d} \delta$

Les points matériels $Q$ de la section droite initiale, supposée circulaire, peuvent 
être repérés par leurs coordonnées polaires initiales : $Q(r, \theta), r \leq R, 0 \leq \theta<2 \pi$ (fig 1).

Une question se pose pour poursuivre une démarche rigoureuse : la section finale contient des points $Q(r, \theta), R<r \leq R$ $+\mathrm{d} R$, inexistants dans l'état initial. Comment alors définir leur position, puis leur déformation, puis leur état de contraintes, dans le problème posé ? L'objection est levée si l'on schématise le problème en deux étapes :

- une étape de croissance sans variation de chargement, de $R$ à $R+\mathrm{d} R$;

- une étape de chargement sans croissance secondaire, sur la section finale de rayon $R+\mathrm{d} R$.

La continuité du phénomène reste respectée dès lors que l'on s'intéresse à un intervalle de temps $\mathrm{d} t$ suffisamment petit.

L'analyse de l'étape de chargement devient alors un problème classique de résistance des matériaux. Le chargement induit aux points $Q(r, \theta)$ un incrément de contraintes (3), linéaire en fonction de la coordonnée radiale $r$ :

$$
d \sigma_{L L}(t, Q)=\frac{d N}{s}+\frac{d M_{x}}{1} y-\frac{d M F_{y}}{1} x
$$

avec $S=\pi(R+\mathrm{d} R)^{2}=\pi R^{2}$, aire de la section droite; $I=\pi / 4(R+\mathrm{d} R)^{4}=\pi / 4 R^{4}$, inertie diamétrale de la section droite; $x=r$ $\cos \theta$ (resp $y=r \sin \theta$ ), distance entre le point $Q$ et l'axe $y$ (resp l'axe $x$ )

soit, en utilisant (2):

$$
\begin{aligned}
& \mathrm{d} \sigma_{L L}(t, Q)=-\frac{\mathrm{d} P}{s} \\
& +\mathrm{r} \frac{-\cos (\delta-\theta)(e \mathrm{~d} P+P \mathrm{~d} \theta)+P e \sin (\delta-\theta) \mathrm{d} \delta}{1}
\end{aligned}
$$

$=-\frac{\mathrm{d} P}{S}-r \frac{\mathrm{d}[P e \cos (\delta-\theta)]}{1}$

L'analyse de l'incrément de déformations $d \varepsilon_{L L}$, qui conduit à celle de l'incrément de courbure et donc à la description cinématique des formes successives prises par la tige en croissance sous son poids propre a été exposée par ailleurs (Schaeffer, 1990; Castera et Fournier, 1990). Elle ne sera pas développée ici.

Le rayon $R(t)$ est caractéristique de l'âge de la section droite; il s'agit donc d'une fonction bi-univoque de l'instant $t$. Par la suite, la date $t$ sera donc repérée par le rayon $R(t)$ et nous écrirons ainsi $\mathrm{d} \sigma_{L L}(R, Q)$ plutôt que $\mathrm{d} \sigma_{L L}(t, Q)$.

\section{ÉTAT MÉCANIQUE DE LA SECTION DROITE À LA DATE $R_{f}$}

La question posée est maintenant : quel est l'état des contraintes $\sigma_{L L}(Q)$, dû au support du poids propre, en un point $Q(r, \theta)$ de la section droite, à l'instant actuel où $R=R_{f}$ ?

\section{Une expression $\sigma_{L L}(r, \theta)$ qui ne tient pas compte de la croissance radiale}

Supposons que le poids $P_{f}=P\left(R_{f}\right)$, excentré de $e_{f}=e\left(R_{f}\right)$ dans la direction $\delta_{f}=\delta\left(R_{f}\right)$, se rajoute intégralement à l'instant final sur la section déjà formée, de rayon $R_{f}$ (d'aire $S=S_{f}$ et d'inertie $l=l_{f}$ ). Le champ des contraintes $\sigma_{L L}(r, \theta)$ est donné par la formule classique de la résistance des matériaux, analogue de (3) sans symbole différentiel :

$\sigma_{L L}(r, \theta)=\frac{-P_{f}}{S_{f}}-r \cos \left(\delta_{f}-\theta\right) \frac{P_{f} e_{f}}{l_{f}}$ 
Cette expression (4) est linéaire en $r$, les tensions et compressions maximales étant atteintes à la surface $r=R_{f}$, respectivement en $\theta=\delta_{f}+\pi$ et $\theta=\delta_{f}$. Elle fait apparaître un secteur comprimé centré sur $\theta$ $=\delta_{f}$, la contrainte de compression maximale étant atteinte à la surface $r=R_{f}$ en $\theta$ $=\delta_{f}$ opposé à un secteur tendu centré sur $\theta=S_{f}+\pi$, la contrainte de tension maximale étant atteinte à la surface $r=R_{f}$ en $\theta$ $=S_{f}+\pi$. Dans le but de fixer des ordres de grandeurs des contraintes envisagées, elle est représentée sur la figure 2, le long de l'axe de tension-compression maximales, et le long de la circonférence à la surface, pour un jeu de données réalistes $\left(R_{f}=15 \mathrm{~cm}, P_{f}=10000 \mathrm{~N}, e_{f}=1 \mathrm{~m}\right)$.

Cette expression (4), correcte lorsque l'on s'intéresse à l'effet d'un effort instantané à l'échelle de la croissance de l'arbre (masse de neige, de givre ..., suppression du poids propre (Fournier et al, 1990), est bien entendu fictive lorsque l'on cherche à représenter l'effet du support du poids propre. Elle servira par la suite de référence.

\section{Prise en compte de la croissance radiale}

Le point matériel $Q$ a été créé, libre de contraintes, à l'instant $t_{r}$ où la section droite avait le rayon $R=r$. II a alors subi des variations infinitésimales d'état mécanique, depuis cet instant initial, jusqu'à l'instant actuel $t_{f}$ où $R=R_{f}$. En utilisant les résultats du paragraphe précédent, il est donc le siège d'un état de contrainte $\sigma_{L L}$ (Q) tel que :

$$
\sigma_{L L}(Q)=\int_{r}^{R_{f}} d \sigma_{L L}(R, Q)
$$

Soit, d'après (3),

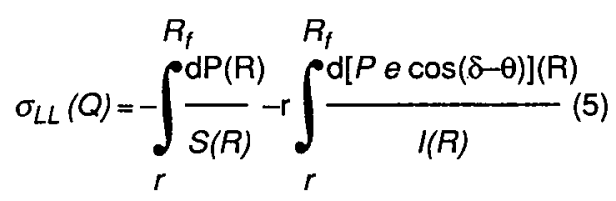

Cette dernière expression (5) appelle quelques commentaires : pour modéliser
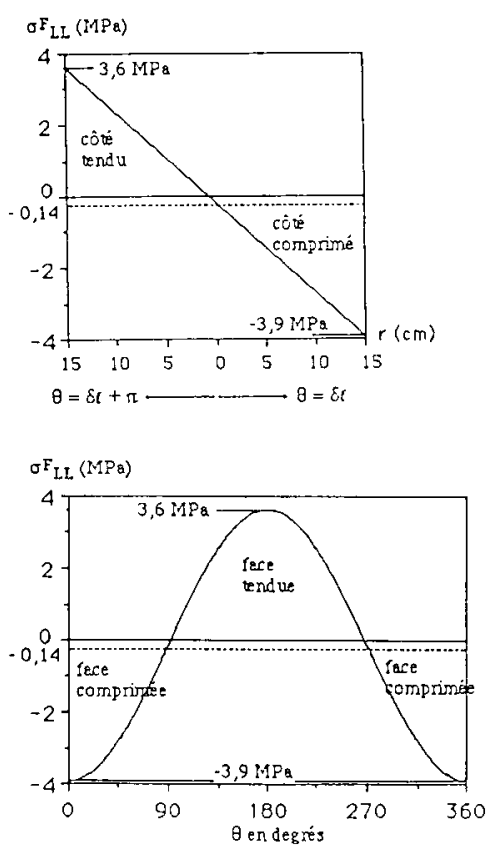

Fig 2. État des contraintes dans un tronc (non croissant) de dimensions données $R f$, initialement non chargé, et subitement soumis à l'action de l'intégralité du poid $P f$ excentré $\left(e_{i}, \delta_{f}\right)$. Les ordres de grandeur découlent d'un jeu de données réalistes $\left(R_{f}=15 \mathrm{~cm}, \mathrm{P}_{f}=10000 \mathrm{~N}, e_{f}\right.$ $=1 \mathrm{~m}$ ). La contrainte longitudinale $\sigma F_{L L}$ est représentée en fonction de la position radiale, le long de l'axe de tension-compression maximale $\left(\theta=\delta_{f}+\pi, \theta=\delta_{f}\right)$, ainsi qu'à la surface $r=R_{f}$. en fonction de la position angulaire $\theta$ sur la circonférence. 
la croissance secondaire, nous avons été amenés à raisonner "pas à pas" pour tenir compte d'une part, des variations de la section porteuse (les caractéristiques géométriques $S$ et / augmentent dans le temps et sont donc des fonctions de $R$ ) et d'autre part, de l'âge différent des points situés sur un rayon de la section droite (la borne initiale de l'intégrale est la date de création $r$ du point $Q(r, \theta)$, et non un instant initial fixe pour les points $Q$.

Le coefficient $\int_{r}^{R_{f}} \frac{\mathrm{d}[P e \cos (\delta-\theta)](t)}{\mathrm{I}(t)}$

dépend de $r$, la distribution de $\sigma_{L L}$ exprimée par (5) n'est donc pas linéaire en $r$. En particulier, la valeur maximale de $\sigma_{L L}$ n'est pas atteinte en un point $r=R_{f}$ de la surface finale comme dans l'expression (4) : en ces points, la date de création $r$ de ces points est l'instant final $R_{f}$, et donc $\sigma_{L L}$ est nul. Les points de la surface, créés tout récemment, ne supportent que l'incrément de chargement ajouté depuis leur création, et donc une part infinitésimale du chargement total.

L'expression (5) montre que la répartition des contraintes $\sigma_{L L}(r, \theta)$ à l'instant final $R_{f}$ dépend de la cinétique de $P(R)$, $e(R)$ et $\delta(R)$, et non des seules grandeurs $P_{f}, e_{f}, \delta_{f}$ identifiables à l'instant final. De fait, des exemples très simples (Fournier, 1990) montrent à l'évidence que la répartition des efforts dans un solide chargé en cours de fabrication dépend des conditions relatives d'élaboration et de chargement.

\section{DONNÉES NÉCESSAIRES \\ À L'ANALYSE DES CONTRAINTES DE SUPPORT}

La formulation du problème requiert donc de se donner les lois d'évolution $P(R), e(R)$ et $\delta(R)$.

\section{Poids $P(R)$}

Les résultats généraux d'études de biomasse (Pardé, 1980; Pardé et Bouchon, 1988) nous poussent à retenir des lois puissance :

$$
P(R)=P_{f}\left(\frac{R}{R_{f}}\right)^{b}
$$

avec approximativement, $2 \leq b \leq 3$

où $P_{f}$ est le poids total supporté dans l'état final, et $b$ un paramètre caractéristique de la cinétique de mise en place du poids propre supporté $: b=2$ signifie que l'arbre supportait déjà le quart de son poids $(P=$ $\left.P_{f} / 4\right)$ à la moitié de son diamètre final $(R=$ $\left.R_{f} / 2\right)$, alors que $b=3$ signifie que la masse supportée s'est accrue plus tardivement puisque à $R=R_{f} / 2, P=P_{f} / 9$.

\section{Lois $e(R)$ et $\delta(R)$}

À un instant donné (et notamment à l'instant final), e et $\delta$ sont évaluables, sur des individus de conformation typée, par le relevé de la projection au sol du houppier (Fournier et al, 1990). 
Les lois d'évolution de l'excentricité du houppier, fonction de l'environnement et du programme génétique de l'arbre, ne sont pas encore, à notre connaissance, des grandeurs couramment manipulées. Quelques principes généraux permettent toutefois d'imaginer qualitativement des scénarios types.

Schématisons tout d'abord l'arbre par un simple mât encastré qui croît en diamètre, en supportant, à la hauteur $H$, une masse concentrée $P(R)$ qui croît avec $R$. Les caractéristiques du "bras de levier" $\mathrm{e}(R)$ et $\delta(R)$ sont donnés entre $R_{i}$ et $R_{f}$, dans chaque section et à chaque pas $R$, par la détermination du torseur des efforts répartis sur la structure en tenant compte de ses déformées successives. Partant d'une situation légèrement déséquilibrée $e_{i}(R)$ petit et $\delta_{j}(R)$ proche de $\pi / 2$, à chaque instant $R$, puisque la masse supportée augmente, les signes de l'incrément de courbure et de la rotation autour de $z$ sont déterminés et conduisent automatiquement à augmenter le déséquilibre.

Selon ces principes, le déséquilibre initial d'une tige ne peut que s'aggraver lorsque le poids supporté augmente, avec des risques d'atteindre des situations critiques de flambement (Fournier et al, 1988). Les grandeurs $e(R)$ et $\delta(R)$ seraient donc des fonctions croissantes de $R$, déterminées par la seule action mécanique du poids propre supporté. Cependant, l'arbre, être vivant, ne répond pas à une telle description simpliste : après une éclaircie ou un chablis, il peut occuper l'espace laissé libre en développant ses axes dirigés vers la lumière, et donc accélérer son déséquilibre mécanique. À l'opposé, il peut lutter contre ce déséquilibre par la croissance primaire (par exemple en relayant l'axe leader par un axe secondaire), ou la croissance secondaire : formation de bois de réaction et redressement des tiges (Wardrop, 1965; Hejnowicz, 1967; Wilson et al, 1977 et
1979). L'arbre possède donc une potentialité d'adaptation (phototropisme ou gravitropisme), sa forme n'est alors plus uniquement régie par de simples lois physiques "passives", et la description mécanicienne doit intégrer les réactions "actives" de la croissance (Fournier, 1989; Castera et Fournier, 1990). Des scénarios où une tige stoppe son déséquilibre ou même se redresse (où le sens de variation de $e(R)$ et $\delta(R)$ change de signe) sont donc tout à fait réalistes.

Les situations schématiques suivantes seront donc étudiées, à partir de lois puissance.

- cas (a) de l'arbre parfaitement symétrique :

$$
e(R)=0
$$

- cas (b) d'un arbre dont le déséquilibre $e$ $(R)$ s'accroît, plus ou moins vite, dans un plan fixe $\delta_{f}$ :

$$
e(R)=e_{f}\left(\frac{R}{R_{f}}\right)^{e x} \quad \delta(R)=\delta_{f}
$$

où $e_{f}$ est la valeur de l'excentricité du centre de gravité dans l'état final $R_{f}$ et qualifie donc le déséquilibre observable dans l'instant final estimable par des relevés dendrométriques usuels (Fournier et al, 1990). Le paramètre ex, positif, qualifie la vitesse avec laquelle ce centre de gravité s'excentre : à $e_{f}$ constant, ex élevé signifie que l'excentricité $e$ a augmenté tardivement.

- cas (c) d'un arbre qui est d'abord déséquilibré, puis se redresse, dans un plan fixe :

$$
\begin{aligned}
& \delta(R)=\delta_{f} \\
& e(R)=k e_{f}\left(\frac{R}{R_{r}}\right)^{e x_{1}} \text { de } 0 \text { à } R_{r},
\end{aligned}
$$




$$
e(R)=e_{f}\left(\frac{R}{R_{f}}\right)^{e x_{2}} \text { de } R_{r} \grave{a} R_{f}
$$

$R_{r}$ est le rayon où la réaction de l'arbre se manifeste : l'excentricité e croît de 0 à $R_{r}$ puis décroît de $R_{r}$ à $R_{f}$. De $R_{r}$ à $R_{f}$, l'arbre se rééquilibre;

$e x_{1}>0$ qualifie la vitesse avec laquelle l'excentricité $e$ augmente dans la phase initiale; $k>1$ représente l'excentricité maximale du centre de poussée atteinte à $R=$ $R_{r}$, rapportée à l'excentricité finale $e_{f}$ observable à l'instant final.

$k, R$ et $e x_{2}$ ne sont alors pas indépendants

$$
\text { de sorte que ex }=\frac{\log k}{\log \frac{R_{r}}{R_{f}}}, e x_{2}<0 .
$$

Les comparaisons seront effectuées à déséquilibre égal dans un état $R_{i}$ proche de l'état initial (nous choisirons $R_{i}=R_{f} / 20$ ), c'est-à-dire à e $\left(R_{i}\right)=\mathrm{e}_{i}$ constant. Le para-

Log $k e_{f} / e_{i}$

mètre $e x_{1}$ est alors fixé égal à

$$
\log R_{i} / R_{r}
$$

L'évolution géométrique de l'arbre est alors paramétrée par $k$, qui est le rapport entre le déséquilibre final et le déséquilibre maximal à $R=R_{r}: k$ qualifie donc l'intensité du redressement entre $R_{r}$ et $R_{f}$.

- cas (d) d'un arbre dont le déséquilibre $e$ (R) s'accroît, et qui "vrille» progressivement :

$$
\begin{array}{r}
e(R)=e_{f}\left(\frac{R}{R_{f}}\right)^{e x} \\
\delta(R)=\delta_{i}+\left(\delta_{f}-\delta_{i}\right) \frac{R}{R_{f}}
\end{array}
$$

$\delta$ initialement égal à $\delta_{i}$, évolue linéairement vers $\delta_{f}$ dans la situation finale.

Les situations (a), (b), (c), (d) vont donner lieu à des simulations numériques des contraintes $\sigma_{L L}(Q)$. Le dessin schématique de la morphologie de l'arbre et de son évolution, est représenté en regard de chaque simulation (figs 3-6), pour chaque situation.

\section{SIMULATIONS NUMÉRIQUES}

Les distributions de contraintes $\sigma_{L L}(Q)$ sont calculées par l'intégration analytique
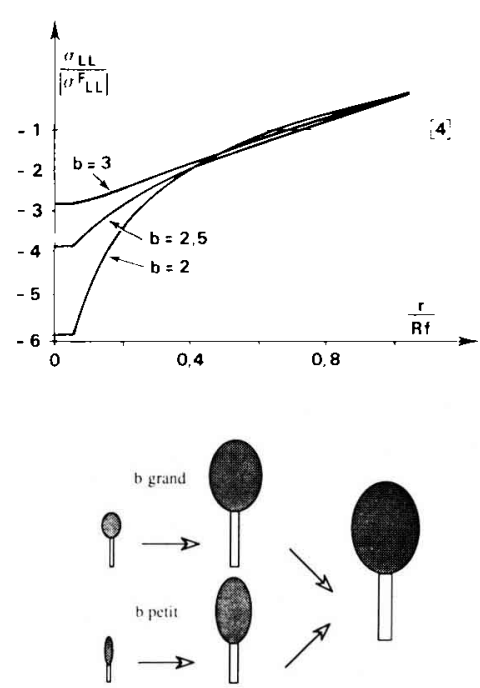

Fig 3. Effet de la mise en place progressive du poids supporté sur la composante longitudinale $\sigma_{L L}$ exercée par ce poids, dans le cas d'un arbre parfaitement symétrique sollicité uniquement en compression. L'unité de $\sigma_{L L}$ est la compression $\sigma_{L L}$ résultant de la distribution uniforme du poids $P_{f}$ sur la section finale. Le calcul est fait pour 3 valeurs : $b=2, b=2,5, b=3$, de la cinétique de mise en place du chargement par rapport à la croissance cambiale $P(R)$, supposée en loi puissance $P(R)=P_{f}\left(R / R_{f}\right)^{b}$, et donc qualifiée par la constante b de la loi puissance. 
ou numérique de (5) dans les différentes situations décrites au chapitre Données nécessaires à l'analyse des contraintes de support, pour diverses valeurs des paramètres $b, e x, k$ (Fournier, 1989). Les résultats sont comparés à $\sigma_{L L}(Q)$ donné par l'expression (4) qui ne tient pas compte de la croissance en raisonnant exclusivement sur la géométrie finale.

\section{Cas (a) de l'arbre \\ parfaitement symétrique}

L'effet du poids se limite alors à une compression $\sigma_{L L}(r)$. La figure 3 représente la distribution $\sigma_{L L}(r)$ pour différentes cinétiques de $P(R)$ schématisées par le paramètre $b$.

$\sigma_{L L}$ rapportée à $\frac{P_{f}}{\pi R_{f}^{2}}$, fonction décroissante

de $r$ nulle à la surface $r=R_{f}$, peut prendre près du cœur des valeurs 3 à 6 fois plus

élevées que la constante $\sigma_{L L}(Q)=-\frac{P_{f}}{\pi R_{f}^{2}}$

prévue par (4). Les valeurs maximales atteintes augmentent quand $b$ diminue, c'està-dire quand la cinétique est telle que le poids supporté par l'individu jeune, de faible section, est plus important.

\section{$P_{f}$}

II reste que — est très faible (de l'ordre de

$$
\pi R^{2}
$$

quelques dixièmes de MPa, cf fig 2). À la suite de Martley (1928), nous conclurons donc que l'effort normal de compression dû au poids propre supporté n'a pas d'effet significatif, même en tenant compte de la croissance radiale.

Dans tout ce qui va suivre, l'effet de compression sera donc négligé devant l'effet de flexion. L'expression (4) s'écrit alors :

$$
\frac{\sigma_{L L}(Q)}{\cos \left(\delta_{f}-\theta\right)}(r)=\frac{-P_{f} e_{f}}{\pi R_{f}^{4 / 4}} r
$$

\section{Cas (b) d'un arbre}

dont le déséquilibre s'accroît, plus ou moins vite, dans un plan fixe

L'arbre restant dans le plan contenant $\delta_{f}$, la distribution $\sigma_{L L}(r, \theta)$ est proportionnelle à
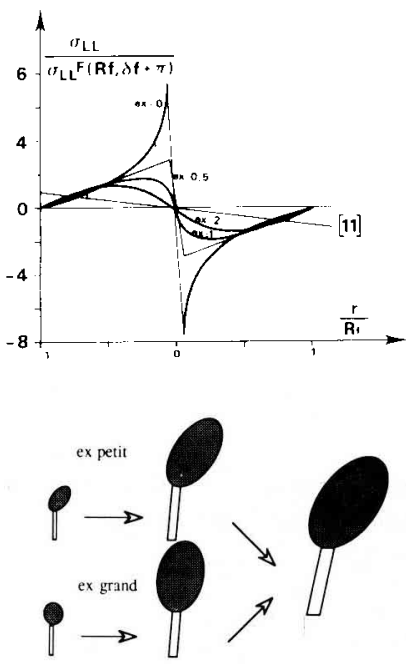

Fig 4. Effet, sur la contrainte longitudinale $\sigma_{L L}$ supportée par le tronc, de la mise en place progressive du poids supporté, pour un arbre qui s'incline de façon monotone dans une direction fixe $\delta_{f}$ L'unité de $\sigma_{L L}$ est la tension maximale $\sigma F_{L L}$ résultant de la distribution linéaire du poids $P f$ sur la section finale (cf fig 2). $\sigma_{L L}$ est représenté le long de l'axe de tension-compression maximale $\left(\theta=\delta_{f}+\pi, \theta=\delta_{f}\right)$. Le calcul est fait pour 4 situations de la cinétique de l'excentrement du poids, qualifiée par la donnée de $e(R)$, supposée en loi puissance $\mathrm{e}(R)=\mathrm{e}_{\mathrm{f}}\left(R / R_{\mathrm{f}}\right)^{\mathrm{ex}}$, et donc par la donnée de ex. Plus ex augmente, plus l'excentricité $e(R)$ s'accroit entre les états initial et final. La cinétique de poids $P(R)$ est choisie constante égale à $P(R)=\mathrm{P}_{\mathrm{f}}\left(R / R_{f}\right)^{2,5}$. 
$\cos \left(\delta_{f}-\theta\right)$. La figure 4 représente les fonctions $\sigma_{L L}(r)$ le long de l'axe $\delta_{f}$,

normées par $\sigma_{L L}\left(R_{f}, \delta_{f}+\pi\right)=\frac{P_{f}, e_{f}}{\pi R_{f}^{3 / 4}}$,

pour $b=2,5$ et différentes valeurs de ex. La comparaison avec la solution (11) montre que les contraintes maximales peuvent être sensiblement plus élevées que la valeur $\sigma_{L L}\left(R_{f}, \delta_{f}+\pi\right)$, qui est la tension maximale prévue par (11), et ne s'exercent pas à la surface $r=R_{f}$, en $\theta=$ $\delta_{f}$ ou $\theta=\delta_{f}+\pi$, mais à une position d'autant plus proche de laxe $r=0$ que ex est petit (donc que l'arbre s'est incliné tôt). $\mathrm{Ce}$ résultat s'explique par une compétition entre l'effet de la flexion, qui induit des contraintes $\sigma_{L L}$ plus importantes à la périphérie, et celui de la croissance, qui veut que les parties internes, existant depuis plus longtemps, soient plus sollicitées. II avait été pressenti par JF Martley à propos de la croissance des branches (Martley, 1928).

\section{Cas d'un arbre qui est d'abord déséquilibré, puis se redresse, dans un plan fixe $\delta_{f}$}

La distribution $\sigma_{L L}(r, \theta)$ reste, pour les mêmes raisons qu'au paragraphe précédent, proportionnelle à $\cos \left(\delta_{f}-\theta\right)$. La figure 5 représente $\sigma_{L L}(r)$ le long de l'axe $\delta_{f}$, normée par $\sigma_{L L}\left(R_{f}, \delta_{f}+\pi\right)$ (cf (11)) pour $b=2,5$, un âge de réaction $R_{r}=R_{f} / 2, e_{i}$ $=e\left(R_{f} / 20\right)=e_{f} / 5$, et différentes valeurs du paramètre de redressement $k: k=1$, ce qui signifie que le déséquilibre $e(R)$ augmente jusqu'à $R_{r}$ puis se stabilise; $k=$ 2 , l'excentricité finale $e\left(R_{f}\right)$ est la moitié de l'excentricité maximale atteinte en $R_{r} ; k=$ 8 , l'arbre s'est considérablement redressé, $e\left(R_{f}\right)$ atteignait 8 fois la valeur $e_{f}$ observable à l'instant final, éventuellement faible.
Il apparaît alors encore plus nettement qu'au paragraphe précédent que la seule observation de la géométrie et de la masse finales ne donne aucune indication sur l'allure de la distribution des contraintes de support de flexion dans l'arbre, puisqu'un arbre aujourd'hui redressé peut comporter des parties internes beaucoup plus tendues et comprimées
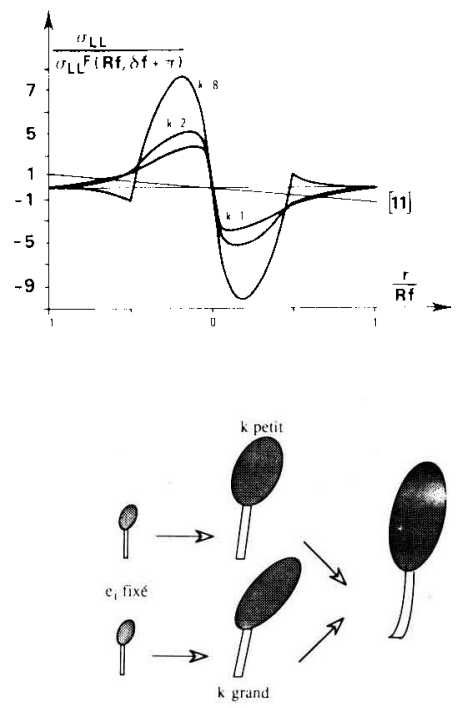

Fig 5. Effet, sur la contrainte longitudinale $\sigma_{L L}$ supportée par le tronc, de la mise en place progressive du poids supporté, pour un arbre qui $s^{\prime}$ incline puis se redresse dans une direction fixe $\delta_{+}$L'unité de $\sigma_{L L}$ est la tension maximale $\sigma_{L L}$ résultant de la distribution linéaire du poids $P_{f}$ sur la section finale ( $c f$ fig 2). $\sigma_{L L}$ est représentée le long de l'axe de tension-compression maximale $\left(\theta=\delta_{f}+\pi, \theta=\delta_{f}\right)$. Le redressement étant supposé commencer à la moitié du diamètre final $(R=$ $R_{f} / 2$ ), le calcul est fait pour 3 situations de la cinétique d'intensité de redressement, qualifiée par la donnée de $k$, rapport entre l'excentricité maximale $e\left(R_{f} / 2\right)$ et l'excentricité finale $e_{f}$ Plus $k$ augmente, plus l'amplitude du déséquilibre, stoppé à $R=R_{f} / 2$, puis redressé jusqu'à la valeur finale $e_{f}$ est importante. Les cinétiques de l'excentrement du poids sont supposées en loi puissance, ainsi que la cinétique du poids $P(R)$ (constante égale à $P(R)=P_{f}\left(R / R_{f}\right)^{2,5}$. 
que ne le laisserait supposer son faible déséquilibre actuel. Un cas de redressement très rapide $(k=8)$ peut même conduire à une situation où le moment $P e$ décroît entre $R_{r}$ et $R_{f}$ car l'effet de réorientation (décroissance de $e(R)$ ) l'emporte sur l'accroissement de masse (croissance de $P(R))$ : le résultat apparemment surprenant est alors que bien que l'arbre final penche dans la direction $\delta_{f}$, l'effet du support du poids propre est de tendre les points proches de la surface dans cette direction $\delta_{f}$ (et de comprimer les points opposés).

\section{Cas (c) d'un arbre dont le déséquilibre s'accroît, et qui vrille progressivement de $\delta_{j}$ à $\delta_{f}$}

La direction du moment fléchissant appliqué est alors progressivement déviée : initialement dans le plan $\left(z, \delta_{i}\right)$, les flexions successives appliquées à l'arbre évoluent pour atteindre le plan $\left(z, \delta_{f}\right)$. Le champ $\sigma_{L L}(Q)$ n'est plus alors proportionnel à $\cos \left(\theta-\delta_{f}\right)$. La fonction $\sigma_{L L}(r, \theta)$ est calculée par l'intégration numérique de (5) pour $b=2,5$, ex $=1$, et $\delta_{i}-\delta_{f}=-\pi / 4$; elle est représentée sur la figure 6 , dans le plan et en coupe dans les directions $\delta_{i}$ et $\delta_{f}$, où elle est superposée aux distributions $\sigma_{L L}$ $(Q)$, ainsi qu'aux distributions du cas précédent obtenues pour $\delta$ constant égal à $\delta_{\psi}$

Lorsque la direction du moment fléchissant appliqué par le poids propre tourne avec la croissance de l'arbre, l'angle polaire définissant la position de la contrainte de compression maximale n'est pas localisé en $\delta=\delta_{f}$, mais s'étale entre $\delta_{i}$ et $\delta_{f}$, d'une façon qui dépend de la cinétique de mise en place du moment appliqué. Plus $b+$ ex sera faible, plus le moment supporté $P e=P_{f} e_{f} \quad\left(R / R_{f}\right)^{b+e x}$ sera élevé sur l'individu jeune, et donc plus cet angle se rapprochera de $\delta_{i}$ en s'éloignant de $\delta_{f}$.
Dans toutes ces simulations, la croissance a été supposée conserver une symétrie de révolution, sans excentrement de la moelle, qui reste par conséquent la fibre neutre (en négligeant l'effort de compression). Une modélisation tenant compte de cet éventuel excentrement a été développée (Fournier, 1989; voir chapitre cas d'un arbre qui est d'abord déséquilibré, puis se redresse dans un plan fixe), qui
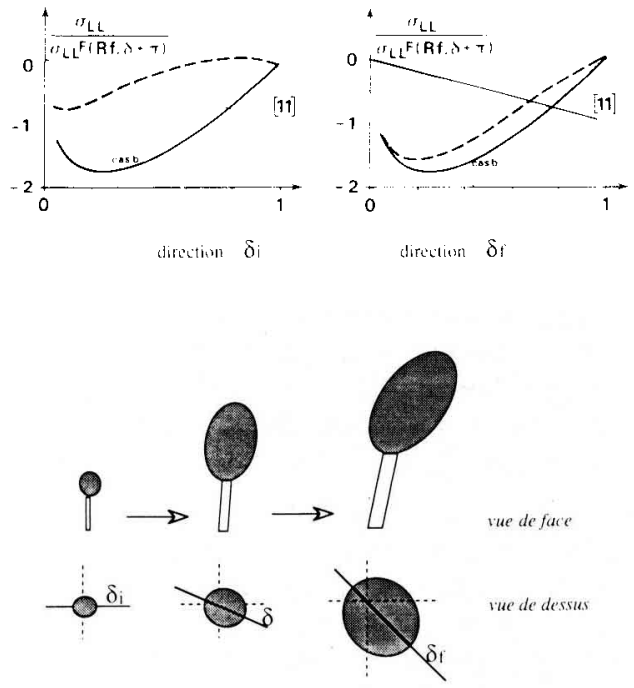

Fig 6. Effet sur la contrainte longitudinale $\sigma_{L L}$ supportée par le tronc, de la mise en place progressive du poids supporté pour un arbre qui s'incline de façon monotone dans une direction variable $\delta_{f}=\delta(R)=\delta_{i}+\left(\delta_{r}-\delta_{i}\right) R / R_{f}$. Le calcul est fait pour $\delta_{f}-\delta_{i}=\pi / 4$. L'unité de $\sigma_{L L}$ est la tension maximale $\sigma_{L L}$ résultant de la distribution linéaire du poids $P_{f}$ sur la section finale, représentée en trait fin (11). $\sigma_{L L}$ est représenté dans le plan de la section droite, ainsi qu'en coupe dans les direction $\delta_{i}$ et $\delta_{f}$ en fonction de la position radiale $r$. La courbe calculée est figurée en pointillés et comparée à celle (en trait continu) où l'arbre s'incline dans une direction fixe (cas b et fig 4). La cinétique de l'excentrement $e(R)$ du poids est supposée égale à $e(R)=e_{f}\left(R / R_{f}\right)$ celle du poids $P(R)$ est supposée égale à $\mathrm{P}(\mathrm{R})=$ $P_{f}\left(R / R_{f}\right)^{2,5}$. 
montre alors un déplacement de cette fibre neutre vers la direction de croissance rapide, sans toutefois atténuer l'effet essentiel du chargement évolutif qui veut que les points proches de la moelle, donc les plus âgés, restent plus sollicités que les points proches de la surface.

\section{CONCLUSION}

L'état mécanique d'une tige à un instant actuel donné dépend non seulement des conditions actuelles de géométrie et de chargement, mais surtout de toute I'histoire de l'évolution de ce chargement. En particulier, les champs de contraintes Iongitudinales $\sigma_{L L}$ exercées par le support de poids propre dans l'arbre déséquilibré ne sont pas une tension ou une compression $\sigma_{L L}$ maximale à la surface, dans l'axe du déséquilibre actuel, car les points proches du cambium, créés récemment, ne sont a priori que très peu sollicités.

Ces champs de contraintes $\sigma_{L L}$ ont alors été simulés dans une section droite, dans diverses situations schématiques de croissance et d'évolution conjointe du chargement, qui confirment quantitativement ce résultat. Les zones les plus sollicitées sont en effet toujours à proximité de la moelle, là où le bois est âgé. Les valeurs maximales de tension ou compression peuvent être sensiblement plus élevées que celles prédites par la distribution statique $\sigma_{L L}$. Elles ne sont pas nécessairement dans l'axe $\delta_{f}$ du déséquilibre final. Prendre en compte les seules grandeurs globales observables dans l'état final ne permet donc pas de décrire l'état mécanique interne d'une tige dû au support du poids propre.

L'analyse mécanique pas à pas proposée synthétise les considérations de certains auteurs (Martley, 1928; Archer et Byrnes, 1974; Archer, 1986; Schaeffer,
1990); pour ne pas compliquer le formalisme de l'exposé, elle a été présentée ici dans le cadre de l'étude d'un chargement particulier, le poids propre de la structure, mais reste généralisable à d'autres sollicitations, telles que la maturation (Fournier, 1989). Elle s'applique donc d'une part, à la description des formes successives prises par un axe en croissance, problème non développé ici (De Reffye, 1979; Schaeffer, 1990; Castera et Fournier, 1990) et d'autre part, à la description des efforts internes permanents et évolutifs supportés localement par le bois d'une tige, les contraintes de croissance, qui sont la superposition des contraintes de support étudiées ici et des contraintes de maturation (Bordonné et al, 1987). Ce phénomène sera étudié globalement dans une publication ultérieure, ce qui permettra d'envisager la validation expérimentale du modèle; en effet, dans l'arbre, les effets du support et de la maturation sont simultanés et indissociables (Fournier, 1989).

Dans tous les cas, l'analyse réclame la reconstitution de l'histoire de la géométrie et des sollicitations de l'arbre. II faut donc s'attacher à dégager, dans la situation actuelle, les paramètres morphologiques et anatomiques pertinents, indicatifs des situations antérieures.

\section{RÉFÉRENCES}

Archer RR (1986) Growth stresses and strains in trees ( $\mathrm{E}$ Timell, ed) Springer series in wood science, Springer Verlag, Berlin

Archer RR, Byrnes FE (1974) On the distribution of tree growth stresses. Part I: an anisotropic plane strain theory. Wood Sci Technol 8, 184-196

Bordonne PA, Okuyama T, Yamamoto $\mathrm{H}$, Iguchi $M$ (1987) Relationships between growth stresses and microfibril angle in the cell wall. In : Colloque IAWA-UIFRO du 15 déc 1987 au CTBA, Paris 
Castera $P$, Fournier M (1990) Aspects mécaniques de la croissance d'un rameau. Colloque Sciences et Industries du Bois 14-15 mai 1990 à Bordeaux, Arbora, Bordeaux, tome 2, 353-364

Edelin C (1989) Données fondamentales d'architecture des plantes. In : Premier Séminaire "Architecture, structure, mécanique de l'arbre", Montpellier, février 1989, LMGMC, USTL Montpellier

Fisher J, Wassmer Stevenson I (1981) Occurrence of reaction wood in branches of dicotyledons and its role in tree architecture. Bot Gaz 142 (1), 82-95

Fournier M (1989) Mécanique de l'arbre sur pied. Maturation, poids propre, contraintes climatiques dans la tige standard. Thèse de l'INP de Lorraine, 164-170

Fournier M (1990) L'arbre, structure en croissance dans le champ de pesanteur : contraintes de support. In : Deuxième Séminaire "Architecture, structure, mécanique de l'arbre", Montpellier, février 1990, LMGMC, USTL Montpellier

Fournier M, Langbour P, Guitard D, Bordonne PA, Sales C (1988) Fast growth species: strains and stresses in a living tree stem. Voluntary Paper, In: IUFRO/P501 Conference on Properties and utilization of fast growth species planted in tropical areas, 15-20 mai 1988, Sao Paulo, Brésil

Fournier M, Langbour P, Guitard D (1990) Mécanique de l'arbre sur pied : les relevés dendrométriques classiques pour quantifier les efforts gravitationnels supportés par un tronc - leurs limites. Ann Sci For 21, 565-577

Guitard D, Fournier M (1989) Éléments de mécanique des solides déformables en vue d'une application à la mécanique de l'arbre sur pied. In:Premier Séminaire "Architecture, structure, mécanique de larbre", Montpellier, janvier 1989, LMGMC, USTL Montpellier

Hejnowicz Z (1967) Some observations on the mechanism of orientation movement of woody stems. Am J Bot 54 (6), 684-689

Martley JF (1928) Theoretical calculation of the pressure distribution on the basal section of a tree. Forestry 2, 69-72

Laroze S (1980) Résistance des matériaux et structures. In: Tome 2 : Théorie des poutres. Eyrolles-Masson, Paris

Parde J (1980) Forest biomass. For Abstr 41, 8, 341-362

Parde J, Bouchon J (1988) Dendrométrie. $2^{\mathrm{e}}$ édn, ENGREF, Nancy

Reffye De P (1979) Modélisation de l'architecture des arbres par des processus stochastiques. Simulation spatiale des modèles tropicaux sous l'effet de la pesanteur. Application au Coffea robusta. Thèse docteur es science, univ Paris Sud Orsay 2193

Schaeffer B (1990) Forme d'équilibre d'une branche d'arbre. C R Séances Acad Sci 311, Sér II, 37-43

Wardrop $A B$ (1965) The formation and function of reaction wood. In: Cellular ultrastructure of woody plants. Proceedings of the advanced science seminar (WA côté, ed) New York

Wilson BF, Archer RR (1977) Reaction wood: induction and mechanical action. Ann Rev Plant Physiol 28, 24-33

Wilson BF, Archer RR (1979) Tree design: some biological solutions to mechanical problems. Bioscience 29, 5, 293-298 